\title{
Roles of Engineering Correlations in Hypersonic Entry Boundary Layer Transition Prediction
}

\author{
Charles H. Campbell ${ }^{1}$ \\ NASA Johnson Space Center, Houston, TX 77058 \\ Rudolph A. King², Michael A. Kegerise 3 , Scott A. Berry ${ }^{4}$, Thomas J. Horvath ${ }^{5}$ \\ NASA Langley Research Center, Hampton, VA 23681
}

$\mathrm{E}$

fforts to design and operate hypersonic entry vehicles are constrained by many considerations that involve all aspects of an entry vehicle system. One of the more significant physical phenomenon that affect entry trajectory and thermal protection system design is the occurrence of boundary layer transition from a laminar to turbulent state. During the Space Shuttle Return To Flight activity following the loss of Columbia and her crew of seven, NASA's entry aerothermodynamics community implemented an engineering correlation based framework for the prediction of boundary layer transition on the Orbiter. The methodology for this implementation relies upon the framework of correlation techniques that have been in use for several decades. What makes the Orbiter boundary layer transition correlation implementation unique is that a statistically significant data set was acquired in multiple ground test facilities, flight data exists to assist in establishing a better correlation and the framework was founded upon state of the art chemical nonequilibrium Navier Stokes flow field simulations. The basic tenets that guided the formulation and implementation of the Orbiter Return To Flight boundary layer transition prediction capability will be reviewed as a recommended format for future empirical correlation efforts. The validity of this approach has since been demonstrated by very favorable comparison of recent entry flight testing performed with the Orbiter Discovery, which will be graphically summarized. These flight data can provide a means to validate discrete protuberance engineering correlation approaches as well as high fidelity prediction methods to higher confidence. The results of these Orbiter engineering and flight test activities only serve to reinforce the essential role that engineering correlations currently exercise in the design and operation of entry vehicles. The framework of information related to the Orbiter empirical boundary layer transition prediction capability will be utilized to establish a fresh perspective on this role, to illustrate how quantitative statistical evaluations of empirical correlations can and should be used to assess accuracy and to discuss what the authors' perceive as a recent heightened interest in the application of high fidelity numerical modeling of boundary layer transition. Concrete results will also be developed related to empirical boundary layer transition onset correlations. This will include assessment of the discrete protuberance boundary layer transition onset data assembled for the Orbiter configuration during post-Columbia Return To Flight. Assessment of these data will conclude that momentum thickness Reynolds number based correlations have superior coefficients and uncertainty in comparison to roughness height based Reynolds numbers, aka $\mathrm{Re}_{\mathrm{k}}$ or $\mathrm{Re}_{\mathrm{kk}}$. In addition, linear regression results from roughness height Reynolds number based correlations will be evaluated, leading to a hypothesis that non-continuum effects play a role in the processes associated with incipient boundary layer transition on discrete protuberances.

\author{
${ }^{1}$ Aerospace Engineer, Applied Aeroscience and CFD Branch, M/C EG3, AIAA \\ Associate Fellow \\ ${ }^{2}$ Aerospace Engineer, Flow Physics Branch, MS ??, AIAA Senior Member \\ ${ }^{3}$ Aerospace Engineer, Flow Physics Branch, MS ??, AIAA Senior Member \\ ${ }^{4}$ Aerospace Engineer, Aerothermodynamics Branch, M/S 408A, AIAA Associate \\ Member \\ ${ }^{5}$ Aerospace Engineer, Aerothermodynamics Branch, MS ??, AIAA Senior Member
}




\section{Introduction}

Successful design and operation of entry spacecraft requires significant interaction between various communities. The technical expertise required encompasses the guidance and control, navigation, aerodynamics, thermal protection system (TPS), aerothermodynamics, structures and other disciplines. Although the risk encountered by an entry vehicle and crew is minimized by some people, the fact remains that the large energy imparted to orbiting spacecraft during launch and ascent is not dissipated until entry. The consequences for failing to embrace this fact include entry vehicle failures, and the significance of the entry regime to safety of flight is a lesson to be learned from the Columbia accident. The reality of these energy levels and their relevance to entry spacecraft is most poignant to the TPS and entry aerothermodynamics communities. Notwithstanding the number of technical communities listed above that must successfully interact on an entry spacecraft design, a large number of specialties exist within the entry aerothermodynamics discipline. These specialties include high temperature gas dynamics, fluid dynamics, computational science, experimental methods, ground and flight test measurement techniques, ground testing, flight testing, chemistry, and others. Each of these specialties have a necessary role in the design and operation of entry vehicles. Within the aerothermodynamics discipline, the phenomenon of Boundary Layer Transition (BLT) is one of the most complex physical processes that is encountered. Because of this complexity, activities associated with BLT investigations require utilization of the talents and experience of many specialties within the aerothermodynamics community. Getting this complexity to reveal its secrets requires concerted effort and determination. In addition, the importance of BLT requires practical methods to be available to the engineering community in order to inform design and operational engineering and the programmatic decision making process. It is within this framework that considerations of the role and value of empirical BLT prediction capabilities are to be considered in this paper.

\section{Background}

The importance of entry BLT prediction can be demonstrated by reviewing the historical utilization of empirical prediction methods. Since hind sight is significantly influenced by the perspectives assembled by experience, and since the Orbiter Return To Flight (RTF) activity was a very significant influence on the authors' combined experience, a background will be provided that is framed by this recent context. Since the Orbiter RTF activity involved development and implementation of an essentially real time entry assessment of TPS damage (Campbell ref, V1 session papers), the goals and implementation were very application oriented.

As an example of this applied focus, it should be pointed out that practicality was of primary significance during the technical activities that occurred as part of RTF efforts following the Columbia accident on February 1, 2003. In particular, 
significant practicality was required for the aerothermodynamic capabilities supporting the Damage Assessment Team (DAT) charter of dispositioning a variety of potential TPS discrepancies identified on-orbit. The types of TPS hardware issues that were expected to occur and do occur on nearly every mission include protruding gap fillers, tile cavities due to ascent debris, out of specification thermal blanket geometry due to the ascent loading environment, etc. The process to provide assessment of these situations requires detailed engineering results to be submitted to the Mission Management Team (MMT) within a matter of, at most, a few days. In concert with the rigor required to review and quality check assessments that have significant implications upon the crew of a mission, these engineering assessments need to be accomplished in a matter of hours. Within the realm of BLT prediction associated with discrete geometries that would promote early transition, the only practical approach for satisfying this engineering challenge was to implement a ground test based empirical correlation applied with a database of Computational Fluid Dynamics (CFD) derived local flow properties at flight conditions (Campbell ref, v1 refs). Accomplishing this required a multi-million dollar activity spanning several years which also required integrating a diverse team of BLT, CFD and experimental experts. In addition this team had to evolve the details of this capability at the same time the broader entry aeothermodynamics, thermal analyses, structural analyses and TPS community was implementing the first ever real time TPS assessment process. The challenges involved in accomplishing this were significant and are too easily dismissed by those unfamiliar with details of the activity. The fact that a highly technical community typically used to time frames of research and design (on the order of months or years) could successfully pull together around this common goal is a testimony to all the individuals involved. In the case of BLT prediction, the outcome of this RTF effort produced an empirically based prediction capability which, in the opinion of the authors, represents the state of the art in discrete protuberance based empirical BLT prediction. Unfortunately, no other BLT prediction capability is currently at a state of maturity that it can substantiate an ability to perform an essentially real time assessment. As a broad engineering and research community, we need a capability to provide higher fidelity BLT prediction capabilities that can be performed on a complete trajectory in a matter of hours. Unless and until something beyond an empirical correlation can accomplish this time frame goal, then engineering practice will continue to utilize an empirical methodology - regardless of the perceived merit or known pitfalls of utilizing a capability that has known and significant uncertainty. Addressing the weaknesses of the current empirical prediction methods by developing and implementing practical high fidelity, mechanism based approaches should be and is a common goal of the engineering, academic and research communities. Conversely, indictment of the weaknesses of current empirical prediction methodologies without open recognition that these are currently the only practical engineering approach is, in the authors opinion, counterproductive.

The practical path of implementation utilized during RTF by the Orbiter entry aerothermodynamics community is, in process, basically the same as that employed 
in the past. Albeit, there have been some key advancements in detail regarding how correlations are selected and a flow field database is constructed. The prevalent use of empirical methods for BLT prediction continues because the complexities associated with BLT prediction have constrained the engineering community for decades. The use of ground based empirical correlations is the common thread running through this history of a practical path, which means providing an answer in the time allotted but with less than desirable accuracy. Other works provide a fairly comprehensive review of various correlations, and the works Schneider (refs) and others (refs) can be reviewed for more background.

Recent Orbiter entry flight tests using a discrete protuberance have been conducted that allow an assessment of the Orbiter RTF empirical BLT approach. These flight test results indicate the validity of fusing empirically based capabilities for design and operation, particularly when they can be flight calibrated. This work will review RTF BLT correlation framework results of the recent Orbiter entry flight experiments on STS-119 and STS-128, together with an investigation of a Knudsen number based correlation. One intent of this discussion will be to characterize an approach for quantitative evaluation of BLT prediction correlations and capabilities using the statistical data as applied for the RTF BLT capability.

Other sections of this paper will provide a perspective on the important role of empirical BLLT prediction in current and historical vehicle design and operation. The practical path mentioned earlier in this introduction and to be discussed more later in this paper requires that engineers have low cost and straight-forward tools to guide decisions with. Such correlation, however, do not by themselves offer a means to probe the mechanisms of BLT onset nor do they provide an apparent path toward improved accuracy and uncertainty. Accomplishing the long sought after goal of mechanism based BLT prediction will lead to a break-through in the aerothermodynamics community's desire to provide more accurate and precise results.

\section{Overview of Historical Empirical BLT Prediction}

The history of entry vehicle operation and design in the United States is replete with examples of the application of empirical BLT prediction. Due to its significance on entry heating, BLT can play a primary role in TPS selection, trajectory shaping and even upon the vehicle configuration. A variety of empirical techniques have been used by design engineers to establish informed inputs to guide design and operational consideration. From the standpoint of the engineering community the fact that empirical BLT prediction has been used almost exclusively is revealing.

It is helpful to reinforce the complete context of BLT prediction, however a full review is beyond the scope of this paper. Instead, some key references and observations will be made together with some discussion of BLT prediction as practiced by the engineering community. A recently published review of blunt body BLT by Schneider, et al. (Wright 32) provides a comprehensive perspective on 
extant flight data. To date, no thorough effort has been performed to assemble the data identified by Schneider with a common analyses approach. Another significant synthesis of data was performed by Reda (wright 43) in an effort to demonstrate the efficacy of $\mathrm{Re}_{\mathrm{k}}$ approaches. However, that work focused the $\mathrm{Re}_{\mathrm{k}}$ family of correlating parameters. An approach focused on investigating a variety of correlating parameters was pursued during the Orbiter Return To Flight (RTF) activity (RTF V1 and V2 references). The benefits derived from considering a comprehensive approach examining multiple correlations must rely upon having a framework to rationally determine the most effective empirical correlation. To accomplish this, the Orbiter RTF aerothermodynamic team utilized a statistical basis to perform this evaluation. More information on this framework can be found in (BLT v1, v2 and King TM).

One key conclusion that has been formulated by the empirical BLT prediction community is the importance of using a common analyses approach and tools. This is especially important when assembling data from a variety of individual sources, but is also essential when formulating a correlation approach for a particular application. As concluded by Berry et al (JSR), the use of different analyses tools can lead to significant bias error affects which will yield differing correlation coefficients and uncertainties. This is a significant conclusion considering that the technical community's goals include developing a consistent means to interpret approaches. As part of formulating the basis of implementation for the Orbiter BLT framework, the conclusion of Berry et. al impacted the approach in a variety of ways. This includes development of a technique of evaluating boundary layer properties with minimal sensitivity to the CFD code utilized, as well as applying a consistent approach across all facilities. Additional information on the computational approach can be found in ( $\mathrm{v} 1$ and v2 references, CFD tool EG notes). In the opinion of the authors, it is also very important that any effort to empirically correlate a body of experimental or flight data need to consider the available suite of correlation parameters. Unfortunately, the history of empirical correlation has seen significant interest by individuals in promoting their correlation without a quantitative comparison basis to make relative accuracy assessments. As a research community, and in particular as design engineers, our common goal should be to have a suite of potential correlations which are then objectively evaluated for accuracy and effectiveness. The goal of such an approach should not be to create a competition, but instead to determine the most reasonable and accurate correlation for each configuration and BLT initiator (e.g. discrete or distributed roughness, crossflow, blowing, etc.). At the same time, investigations into a variety of potential correlations should continue as long as the engineering community makes use of empirical correlations.

A brief summary of BLT prediction for the U.S. human spaceflight vehicles reveals a fairly limited set of information from the Mercury and Gemini programs. Beyond BLT affects upon the afterbody, it appears that not much detailed consideration was given to BLT prediction. Engineers utilized wind tunnel data to gain insight, margin was included in the design and then flight data provided validation of the design. 
Gemini followed a similar approach, but had the advantage of understanding developed from Mercury. Similarly with Apollo, information and insight gained from previous Mercury or Gemini flights were leveraged for the Apollo design. Additional Apollo ground testing was performed to provide configuration specific data. And, ultimately, flight data again provided validation of the design which enabled an engineering level characterization of BLT in the flight regime (ref Apollo CR). This is the heritage of approach that has benefited all entry vehicle design in the U.S., whereby actual flight experience informs each additional increment forward in design. The Orion project is leveraging this heritage from Apollo. However, since the Orion spacecraft is larger than Apollo and flies a different trajectory, Apollo flight data does not provide a comprehensive picture. More details regarding boundary layer transition data from these blunt configurations is identified in Schneider (Wright 38). At this time, the Orion aerothermodynamics community is assuming fully turbulent entry (Ref?) since the marginal weight benefits identifiable with empirical BLT prediction techniques are small. However, the authors that as the Orion design matures and the project begins considering how to improve the capability of an existing design, higher fidelity BLT prediction capabilities will likely be pursued. What capabilities are utilized will, in large part, depend upon the development level and implementation maturity of the techniques available at that time.

Application of empirical BLT prediction to the Orbiter has been consistent over the three decades since this vehicle's conception. Although unlike Mercury, Gemini and Apollo in that these blunt configurations had ablative TPS which was the primary contributor to BLT, the Orbiter design and development team appears to have invested the most significant effort on BLT for any entry vehicle of its day. BLT concerns were extremely important for the Orbiter because of the silica tile based TPS. Steps and gap between neighboring tiles had to be specified and controlled in order to drive the occurrence of BLT to lower entry Mach numbers. Not only did BLT need to be forced to lower Mach numbers for a typical reentry trajectory, but also for the full suite of envisioned DoD and NASA nominal and abort trajectories. Ultimately, the prime contractor (Rockwell International at the time) implemented an empirical BLT correlation consistent with the work of Van Driest and Blumer (Berry 14). Additional perspective on the prime contractor's approach to BLT prediction and control/maintenance of the specification for tile steps and gaps can be found in Haney, et al. (Berry 1). Additional efforts by NASA to utilize empirical approaches can be found in the works of Bouslog, et al (Berry 2 and 4), Berry (Berry 3), Goodrich (??) and Bertin, et al (Berry 29). Efforts in the last several years by the Orbiter engineering community were focused on baselining a state of the art empirical BLT prediction capability (BLT v1 and V2 and King TP/TM) for the Shuttle Program as a result of the Columbia accident on February 1, 2003. Although not described herein in any detail, these historical Orbiter empirical BLT prediction capabilities have been primarily focused on distributed and discrete BLT. The recent Orbiter Return To Flight activities also included efforts to characterize BLT induced by small tile damage due to ascent debris impacts, discrete BLT due to protruding gap fillers or TPS repair hardware in addition to surface blowing due to localized 
ablative repairs. (v1 and v2 refs) One of the most recent efforts of the Orbiter aerothermodynamics team involved defining an approach to better characterize the suppression of wing leading edge turbulence at high Mach numbers due to an upstream BLT event (King TP, Berry 2010 AIAA). Leveraging the work of Poll (Reference), King, et al. implemented an Rbar approach which has been calibrated with limited Orbiter flight and wind tunnel data.

One of the most significant aspects of the Orbiter RTF BLT prediction effort was an emphasis was placed on establishing an experimental data set from which quantitative statistical evaluations could be made using a variety of empirical correlations. In the authors' opinion consideration of a set of experimental data using only a single type or family of empirical correlations will lead to selfreinforcing conclusions about adequacy, accuracy and effectiveness. For the Orbiter RTF activity, the most appropriate and accurate empirical correlation was sought, regardless of its origin. Another important consideration of this activity was its reliance on a common analysis framework. This was done to avoid bias errors and additional uncertainty that can be introduced as a result of using multiple analyses approaches within a common activity, as concluded by Berry, et al (Berry 6).

The configurations discussed above are all U.S. human spaceflight vehicles that became operational, there have obviously been other significant efforts that utilized empirical BLT prediction techniques. Efforts to characterize nosetip transition as part of the Passive Nosetip Transition (PANT) program (Berry 13) represent a significant technical contribution. Stetson et. al (Berry 40) provides a valuable perspective assembling information on blunt body configurations. Schneider (Wright 38) provides an even more comprehensive survey of available BLT data that was motivated in part by efforts to characterize blunt body transition for the Orion crewed capsule. In addition, information on BLT for conical configurations is available in Boudreau (Berry 31).

NASA efforts in the 1990's to develop and fly a variety of entry vehicles also relied on the use of empirical BLT prediction. These include X-33 (Berry 6), X-38 (Berry 7) and X-34 (Berry 20). A synthesis of data in the $\mathrm{Re}_{\mathrm{k}}$ family of correlations (Wright 33) also represents an important contribution because of that work's effort to compile multiple data sets into a common assessment. Other efforts to perform design activities using a simple $\mathrm{Re}_{\theta}$ criterion (Wright 39-40) reinforce the need for straightforward critical design values for vehicle development. In a recent work by Lin et. al (entry vehicle approach) a very important set of information was provided in regard to how empirical BLT prediction and design criterion have been utilized to improve trajectory accuracy for spherically blunted cones. The weakness of using a simple $\operatorname{Re}_{\theta}$ criterion can be seen by reviewing the significant uncertainty demonstrated in data obtained during the SWERVE flight test activity (SWERVE Reference Candler 1). 
Of particular note among U.S. efforts performed in the last several decades is the National AeroSpace Plane (NASP) effort. This activity represents the only historical activity that made used of BLT prediction techniques beyond empirical correlations. Although the actual design selection utilized an $\operatorname{Re}_{\theta}$ basis of prediction based on flight Navier Stokes simulations, significant effort was also invested in using linear stability analyses (Lau NASP, Malik ref from Cander). Although the NASP program was the only historical effort to make use of stability modeling for BLT prediction, it will most certainly not be the last program or project to do so. Recently, successful application of Parabolized Stability Equation (PSE) methods have been used to gain insight into wind tunnel and flight BLT for the Mars Science Lander (Wright 45, 46, 149). In addition, an even more recent effort to perform PSE modeling on the Orbiter at flight conditions (Johnson 2010 Orlando) has been performed. This trend is an encouraging one, as it represents extension of the theoretical framework first defined by T. Herbert (Ref) an application framework that has potential for use by the entry design and development community. Also of significant interest regarding this trend are activities using high fidelity CFD analyses to better characterize discrete protuberance boundary layer transition (other session refs). These efforts are in part being motivated by the Orbiter BLT Flight Experiment, but are also an additional indication that the multiple U.S. government agencies (e.g. DOE, Air Force, NASA) are making investments in the BLT prediction arena.

This section seeks to summarize how empirical BLT prediction is ubiquitous in the vehicle design community. As far as the authors have been able to ascertain, it appears that every significant NASA entry vehicle has utilized some form of empirical BLT approach. A word of caution is warranted in association with the statement to avoid any potential misunderstanding. The conclusion to draw from this statement is not that the BLT community should accept the status quo of empirical BLT correlations. The appropriate conclusion to draw is that design and operational engineers require simple and effective means to rapidly gain knowledge regarding each scenario in order to inform the decision making process. This informed knowledge will be based upon the capabilities currently in existence that can provide answers in the allotted time frame. To put this in another context, a perfect answer obtained after a critical design decision is made, after a vehicle failure or worse yet, loss of a vehicle and crew is simply too late to influence decisions. Engineers and decision makers have to choose a course of action based on what is known at the time, based on the best information that is currently available. By itself, hoping for an improved answer that will come in too late is not a strategy to depend on. In the vernacular of project management, hope is not a management tool. The BLT research and development community needs to strive for tools, techniques and methods that can provide more accurate, high fidelity BLT prediction in a timely and responsive manner. Historically, investment in hypersonic BLT has been cyclical, and it appears that we are currently seeing increasing national investments in this arena. However, if additional engineering capabilities do not materialize in the current cycle of increased support, then the next downturn in support is very likely to be at least as significant as the last cycle. Therefore, one of our overall goals must be to not only perform research that 
develops understanding, but also to accomplish research and development that provide capabilities which the engineering and design communities can use to impact programmatic decisions. More consistent investment and forward progress in BLT research and development depends upon delivering increased understanding as well as providing engineering capabilities that can impact vehicle design and operational decisions.

\section{Quantitative Evaluation of BLT Correlation Accuracy}

Engineering development and design seeks to utilize any method of sufficient accuracy for guiding the decision making process. The methods utilized to guide decisions will be more accurate in some instances and less accurate in others. The importance of engineering approaches are not to be judged by how perfect they are, but instead by their efficacy at impacting a design or operational decision based on an appropriate level of confidence. In the case of BLT correlations, the efficacy, value and utility of engineering correlations has been validated by decades of use. This has been discussed in the previous section where it is pointed out that apparently every U.S. human spaceflight vehicle and many other entry vehicles have made use of empirical BLT prediction. Having the desire for more accurate and precise BLT prediction capabilities must also be accompanied by substantiated accuracy and practical implementations. The implementation selected for a given circumstance must also then be responsive to the applied engineering time frame and cost framework. It is the desire of the authors, and presumably, the hypersonic entry BLT technical community to provide more accurate prediction capabilities. This is a goal that the authors believe is shared across the engineering, research and academic communities. Current engineering practice uses empirically based BLT correlations, however, as embodied in the approach utilized in development and application of the Orbiter entry RTF BLT prediction capability.

The Orbiter RTF entry aerothermodynamics community has implemented a state of the art framework for discrete protuberance based empirical BLT predictions (REFS V1, V2 Berry, King). In addition, using a philosophy and methodology similar to that employed for discrete protuberances, the Orbiter team has also implemented small cavity empirical BLT prediction (REFS) and suppressed wing leading edge or attachment line BLT empirical BLT prediction (REFS). In an effort to communicate more of the basis and philosophical approach behind the role and implementation of these correlations, a review of the discrete protuberance BLT correlations evaluated by the Orbiter team during RTF will be provided. To further clarify this approach, several additional correlations will be assessed to illustrate a recommended process for empirical correlation selection given a body of experimental and flight data.

Discrete protuberance BLT correlations identified and investigated for implementation supporting the Orbiter project during RTF provide a reference for a state of the art empirical framework. Key aspects of this approach involve the following: a) development of a statistically significant experimental database across multiple hypersonic facilities using scale models of the flight configuration, b) 
generation and inclusion of experimental data relevant to the variability of discrete protuberance geometries of interest, c) utilization of flight data to define the effect of ground to flight scaling, d) implementation of an appropriately accurate Navier Stokes flow field database at ground and flight conditions with thermo-chemistry modeling appropriate to the flow physics, and e) unbiased investigation of multiple empirical correlations to define the most accurate correlation for the selected vehicle configuration. Judicious implementation of each of these five tenets form a necessary foundation for generating the results provided in Table 1, as adapted from King TP ref.

The information provided in Table 1 summarizes several statistical values and best fit coefficients for the discrete protuberance BLT empirical correlations investigated by the Orbiter team. In the first row is an equation defining the power-law relationship used to define each correlation. The statistical values provided include the correlation parameter $\mathrm{R}$, a non-dimensional metric of the variation between the $+95 \%$ and $-95 \%$ bounds represented by $\Delta \mathrm{C}_{95} / \mathrm{C}$, and a measure of the tunnel to tunnel variation of the best fit coefficients specific to data from each facility $\sigma_{\mathrm{C}} / \mathrm{C}$. Also included are best fit coefficients of the ground plus flight data ensemble, flight data only best fit coefficient and the ratio of these two. In cases that specific values were not currently available, a (-) has been entered into Table 1.

Table 1. Power Law Protuberance BLT Correlations from Reference (King TP)

\begin{tabular}{|c|c|c|c|c|c|c|}
\hline Power Law Regression & \multicolumn{3}{|c|}{ Ensemble Statistics } & \multirow[b]{2}{*}{$\begin{array}{l}\text { Ensemble Best } \\
\text { Fit Coefficient }\end{array}$} & \multirow[b]{2}{*}{$\begin{array}{l}\text { Flight Best Fit } \\
\text { Coefficient }\end{array}$} & \multirow{2}{*}{$\begin{array}{l}\text { Flight to Ensemble } \\
\text { Correlation } \\
\text { Coefficient Ratio }\end{array}$} \\
\hline Correlation Equation & $\mathbf{R}$ & $\Delta \mathrm{C}_{95} / \mathrm{C}$ & $\sigma_{c} / C$ & & & \\
\hline$\left(\mathrm{Re}_{\theta} / \mathrm{M}_{\text {edge }}\right)(\mathrm{k} / \theta)\left(\mathrm{H}_{\mathrm{e}} / \mathrm{H}_{\mathrm{w}}\right)^{0.44}=\mathrm{C}$ & -0.92 & 1.05 & $15.5 \%$ & 350.3 & 450.7 & 1.29 \\
\hline$\left(\mathrm{Re}_{\theta} / \mathrm{M}_{\text {edge }}\right)(\mathrm{k} / \delta)\left(\mathrm{H}_{\mathrm{e}} / \mathrm{H}_{\mathrm{w}}\right)^{0.30}=\mathrm{C}$ & -0.91 & 1.10 & $19.9 \%$ & 42.8 & 61.3 & 1.43 \\
\hline$\left(\operatorname{Re}_{\mathrm{k}}{ }^{0.6}\right)\left[\left(\operatorname{Re}_{\theta}\right)\left(\mu_{\mathrm{e}} / \mu_{\mathrm{k}}\right)\right]^{0.40}=\mathrm{C}$ & -0.84 & 1.30 & $35.0 \%$ & 217.6 & 396.8 & 1.82 \\
\hline$\left(\mathrm{Re}_{\theta} / \mathrm{M}_{\text {edge }}\right)(\mathrm{k} / \delta)=\mathrm{C}$ & -0.88 & 1.25 & $21.2 \%$ & 31.9 & 41.6 & 1.30 \\
\hline$\left(\operatorname{Re}_{\theta} / \mathrm{M}_{\text {edge }}\right)\left(\mathrm{k} / \delta^{*}\right)=\mathrm{C}$ & -0.87 & 1.32 & $34.9 \%$ & - & - & - \\
\hline$\left(\operatorname{Re}_{\theta} / \mathrm{M}_{\text {edge }}\right)(\mathrm{k} / \theta)=\mathrm{C}$ & -0.86 & 1.36 & $23.3 \%$ & - & - & - \\
\hline$\left(\mathrm{Re}_{\theta} / \mathrm{M}_{\text {edge }}\right)(\mathrm{k} / \delta)\left(\mathrm{T}_{\mathrm{e}} / \mathrm{T}_{\mathrm{w}}\right)^{0.16}=\mathrm{C}$ & -0.89 & 1.20 & $23.6 \%$ & - & - & - \\
\hline$\left(\rho_{k} u_{k} k / \mu_{k}\right)=C^{+}$ & -0.79 & 2.51 & $30.9 \%$ & - & - & - \\
\hline
\end{tabular}

Development of the discrete protuberance BLT empirical correlations and results listed in Table 1 was performed while utilizing the tenets a-e identified earlier in this section. The experimental data acquired on the Orbiter configuration were acquired in the NASA LaRC Mach 6 air, Mach 10 air and Mach 6 CF4 facilities (Berry AIAA, King TP, others). In addition, experimental data on the Orbiter configuration were also obtained in the CUBRC LENS facility at Mach 10,14 and 16. Discrete protuberances were investigated for several protuberance configurations representative of the geometries considered possible for Orbiter TPS (King REF for TEI, Cassady TM). Orbiter flight data for protruding gap fillers were included in the ensemble of experimental data. This flight data also provided the basis for a flight best fit correlation result, hence the values tabulated in the sixth column of Table 1 
for several of the correlations. A Navier Stokes database of Orbiter configuration ground test simulations and flight simulations was developed using thermochemistry appropriate to the facility or flight environment. As demonstrated in Table 1, multiple empirical correlations were investigated that span the potential spectrum of boundary layer flow parameters. Additional correlations were also investigated that are not identified in the RTF BLT team documentation, and are not represented in Table 1. Open minded consideration of different correlations suggested very recently by others in the community (Reshotko personal comm.. $6 / 09$ ) has resulted in other investigations (King TP) of several correlations which utilize $\mathrm{k} / \theta$ in the parameter set. It is also of interest to the authors to investigate utilization of altitude in the correlation parameter set, because of perspective provided by other individuals regarding the efficacy of empirical BLT correlations. (Ref Reshotko - is Re-theta good enough paper) Each of the correlations summarized in Table 1 utilized the statistically significant ensemble results for a ground test program including over one-hundred discrete data points and ten Orbiter flight data points from protruding gap fillers.

Table 1 loosely orders the correlations in accordance with their accuracy, as interpreted by the authors. Of particular note are the second and third correlations from the top, which are the methods baselined in the Orbiter RTF BLT V2 prediction tool for Space Shuttle Program support. The first correlation listed in Table 1 is fairly new and is being evaluated for potential use in support of the Space Shuttle Program, due to its incremental improvement over the correlation in row 2 of Table 1. The accuracy of each correlation is evaluated by considering the three statistical parameters included in Table 1 . The range of correlation values range between 0.79 and 0.92. Considering that engineering practice considers correlation values higher than approximately 0.5 of significance, all of these correlations can be of potential utility to practicing design engineers. What is undesirable about these correlations is the uncertainty values included in the third and fourth columns of Table 1 . For an Orbiter on entry, these uncertainty values lead to predictions of BLT onset that vary by approximately plus/minus two in Mach number. Nonetheless, a ranking of accuracy has been established by considering the three statistical measures included in Table 1. Of additional information is the ratio of best fit correlation coefficients for the ensemble of ground and flight data included in column five and the best fit coefficient of the flight data shown in column six of Table 1 . This ratio provides insight that is very important to note. The magnitude of this ratio is approximately two times the non-dimensional sigma value included in column six for each correlation. These data imply that the ground to flight scaling affect is approximately twice as large as the tunnel to tunnel variability. This scaling ratio can also be assessed in relationship to the variability of the ensemble of data for the second correlation in Table 1 by graphically examining the results shown in Figure 1. Note that the $-95 \%$ correlation line, shown in red, has a value of 35.3 . Also note from Table 1, that the ensemble data best fit coefficient is 42.8. From this result one can conclude that the flight scaling affect is almost as large as the difference between the $95 \%$ and best fit correlation lines. At first glance, one might conclude that this result means that the confidence of a flight scaling affect being the value 
provided in the last column of Table 1 is approximately $95 \%$. However, this is an incorrect conclusion because there is uncertainty in the height determination of the historical Orbiter protruding gap fillers that is comparable to the uncertainty of the ensemble correlation. This effect is something that the Orbiter BLT Flight Experiment team will seek to clarify by obtaining multiple Orbiter flight BLT onset data points with a known geometry. Flight BLT onset data acquired from STS-119 and STS-128 as part of the Orbiter BLT Flight Experiment (Refs Campbell, Anderson) are included in Figure 1. Additional details regarding the flight experiment effort can be found in Anderson (ref) and Spanos (ref). This recent flight data demonstrates good comparison with RTF BLT V2 predictions, with flight BLT occurring well within one sigma uncertainty and at slightly earlier times than predicted. Even with the existing data ensemble uncertainty, it is important to note that all of the correlations included in Table 1 show a consistent trend where the flight environment leads to BLT onset at higher Reynolds numbers or higher discrete protuberance heights than a ground test based empirical correlation will predict. Evaluating the statistical confidence of this observation that there is a flight scaling effect will be important after two additional BLT FE flights are completed in 2010 before the Space Shuttle is retired.

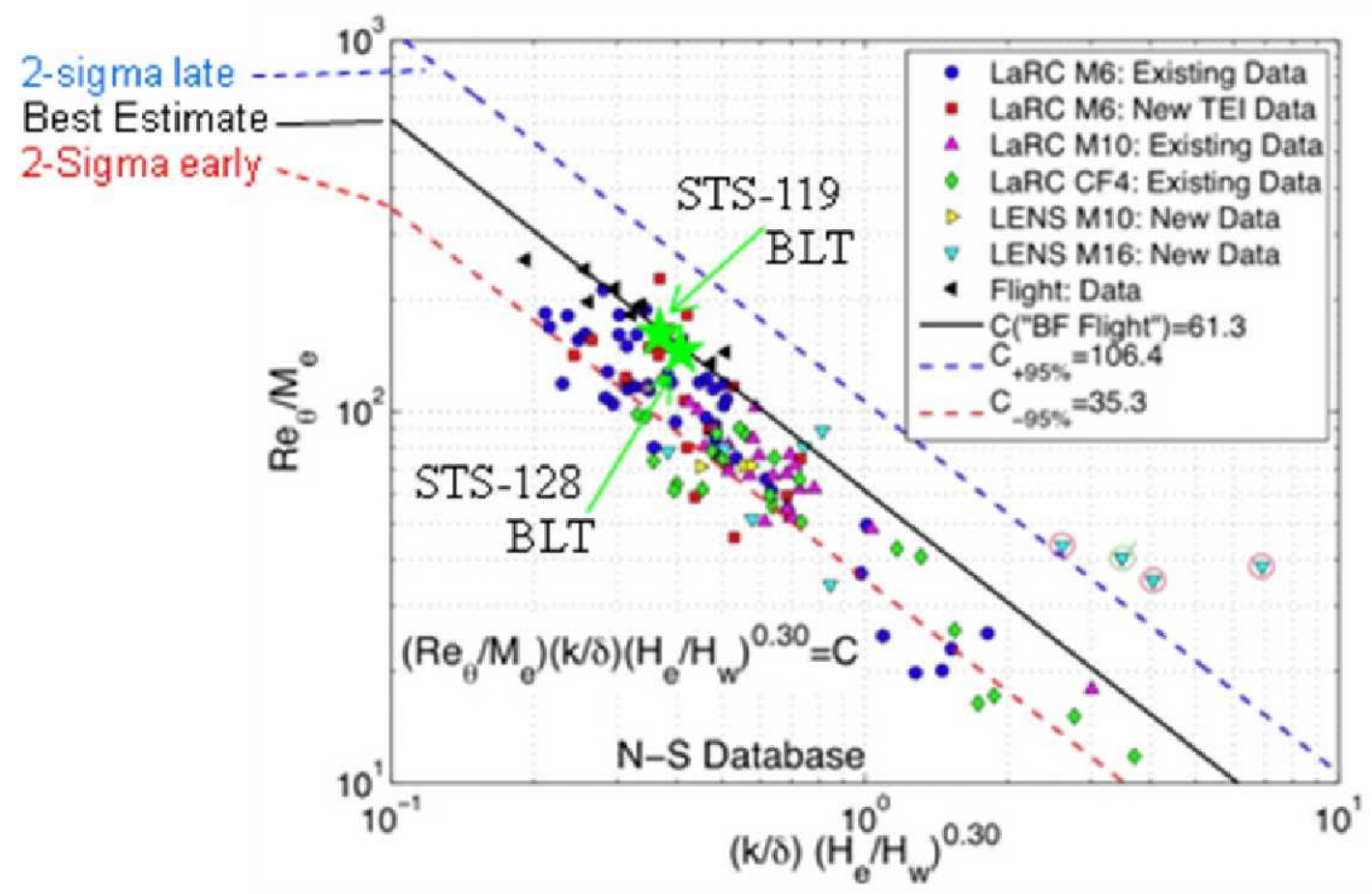

Figure 1. Graphical results for an Empirical BLT Prediction correlation of Orbiter data for protuberances, adapted from Ref. King TP to include BLT Flight Experiment results from STS-119 and STS-128.

There is also another important consideration regarding the magnitude and confidence of a flight scaling effect, as discussed in the previous paragraphs. For an engineer attempting to apply one of these empirically based correlations to a flight 
vehicle, it appears that ground to flight scaling is not insignificant. Thus, in concert with correlation uncertainty and accuracy being critical to an engineer, a bias effect due to flight scaling will also be a critical consideration. A conclusion to draw from this examination of flight scaling is that application of empirical discrete protuberance BLT prediction correlations established only with ground test data will be inaccurate and conservative without sufficient flight data to accurately characterize the flight scaling bias error. Once flight data is acquired, the magnitude of the flight bias error can be evaluated. Depending on the conclusions established from such an assessment, a lower flight bias error for an alternate correlation than what was originally selected pre-flight may subsequently motivate operational selection of a different empirical correlation. This should reinforce to the reader the comments provided earlier in this paper regarding the goal of an engineer is to provide the most reasonable and accurate information to guide decisions. An engineering and technical community should be motivated to change their prediction basis if an improved basis is identified because of new information, whether the source is flight data or because a practical implementation of a new method matures out of the research community. Diligent application of the open minded investigation of alternate frameworks is also one of the tenets outlined earlier that has guided the Orbiter RTF entry aerothermodynamics community, which continues to be utilized by this community.

Let us shift gears now to expanding the characterization beyond power-law correlations as utilized in developing the results provided in Table 1 and Figure 1 . In order to enhance the spectrum of correlations considered, results from linear regression analyses of the Orbiter ground and flight data are included in Table 2. These linear regression results will be used as an example to illustrate how statistical function results can be used to quantitatively rank several possible empirical correlations. The primary benefit of this approach is that it provides a rational and quantitative basis to objectively evaluate options. In addition to using these results as an illustration, several observations will be made regarding the accuracy of the $\operatorname{Re}_{\theta}$ and $\operatorname{Re}_{\mathrm{kk}}$ empirical correlation frameworks.

Several correlations have been selected for evaluation based on similar boundary layer parameters as those utilized in the results included in Table 1. Since linear regression potentially involves multiple correlation coefficients, the matrix of regression coefficient established from linear regression results are included in Table 2. Table 3 contains information similar in format and context to the results included in Table 1. Figures 2 and 3 represent the linear regression results for the first and third correlations included in Tables 2 and 3, and include the best fit correlations for both the ensemble of combined ground and flight data as well as the best fit result for only the flight data. Uncertainty in the linear regression results is characterized by the $\Delta \mathrm{F}_{4 \sigma} / \mathrm{F}_{\text {mean }}$, where $\Delta \mathrm{F}_{4 \sigma}$ is four times the standard deviation of the correlation, and $F_{\text {mean }}$ is the mean value of the correlation function across all data. Since the results included in Table 1 are based on power law correlations, and the results in Tables 2 and 3 are based on linear regression, it should be noted that 
direct comparison of the uncertainty metrics represented by $\Delta \mathrm{C}_{95} / \mathrm{C}$ in Table 1 and $\Delta \mathrm{F}_{4 \sigma} / \mathrm{F}_{\text {mean }}$ in Table 3 is not recommended.

Table 2. Linear Regression Protuberance BLT Correlations

\begin{tabular}{|c|c|c|c|c|c|c|c|}
\hline \multicolumn{2}{|c|}{ Linear Regression Coefficients } & \multirow[b]{2}{*}{$\mathrm{m}_{\mathrm{i}}$} & \multirow[b]{2}{*}{$M_{\text {edge }}$} & \multicolumn{4}{|c|}{$f_{i}$} \\
\hline Correlation & $\mathrm{b}$ & & & $\left(M_{k} / B_{k}\right)$ & $\left(\rho_{k} u_{k} / \rho_{e} u_{e}\right)$ & $(\mathrm{k} / \delta)$ & $\left(\mathrm{H}_{\mathrm{e}} / \mathrm{H}_{\mathrm{w}}\right)$ \\
\hline $\operatorname{Re}_{\theta}=m_{1}\left(M_{\text {edge }}\right)+b$ & 222.23 & & -24.90 & - & - & - & - \\
\hline$\left(\operatorname{Re}_{\theta} / M_{\text {edge }}\right)=b$ & 96.72 & & - & - & - & - & - \\
\hline$\left(\rho_{k} u_{k} k / \mu_{k}\right)=m_{1}\left(M_{k} / B_{k}\right)+b$ & 32.51 & & - & 324.60 & - & - & - \\
\hline \multirow[t]{5}{*}{$\left(\rho_{k} u_{k} k / \mu_{k}\right)=b+m_{i} f_{i}$} & 127.64 & & - & 62.86 & 127.64 & 287.97 & 2.94 \\
\hline & 46.39 & & - & 141.05 & -50.88 & 159.16 & -0.39 \\
\hline & & $n_{j k}$ & & \multicolumn{4}{|c|}{$\mathrm{g}_{\mathrm{jk}}$} \\
\hline & $\left(M_{k} / B_{k}\right)$ & & - & - & 46.39 & -86.56 & - \\
\hline & $\left(\rho_{k} u_{k} / \rho_{e} u_{e}\right)$ & & - & - & - & 215.65 & - \\
\hline$\left(\rho_{k} u_{k} k / \mu_{k}\right)=b+m_{i} f_{i}+n_{j k} f_{j k}$ & $(\mathrm{k} / \delta)$ & & - & - & - & - & - \\
\hline
\end{tabular}

Table 3. Linear Regression Protuberance BLT Correlation Statistics

\begin{tabular}{|c|c|c|c|c|c|c|}
\hline Linear Regression Results & & nble St & & st & st Fit & Flight to \\
\hline Correlation Equation & $\mathbf{R}$ & $\begin{array}{l}\Delta \mathrm{F}_{4 \sigma} / \\
\mathrm{F}_{\text {mean }}\end{array}$ & $\sigma_{\mathrm{c}} / \mathrm{C}$ & $\begin{array}{l}\text { Fit Slope / } \\
\text { Intercept }\end{array}$ & $\begin{array}{l}\text { Slope / } \\
\text { Intercept }\end{array}$ & $\begin{array}{l}\text { Ratio Slope / } \\
\text { Intercept }\end{array}$ \\
\hline $\operatorname{Re}_{\theta}=m_{1}\left(M_{\text {edge }}\right)+b$ & 0.13 & 1.08 & - & $-24.89 / 222.23$ & $-84.25 / 505.25$ & $3.38 / 2.27$ \\
\hline$\left(\operatorname{Re}_{\theta} / M_{\text {edge }}\right)=m$ & - & 2.16 & - & $96.72 /-$ & 191.34 / - & $1.98 /-$ \\
\hline$\left(\rho_{k} u_{k} k / \mu_{k}\right)=m_{1}\left(M_{k} / B_{k}\right)+b$ & 0.67 & 1.99 & - & $324.60 / 32.51$ & 364.62 / 121.92 & $1.12 / 3.75$ \\
\hline$\left(\rho_{k} u_{k} k / \mu_{k}\right)=b+m_{i} f_{i}$ & 0.88 & 1.27 & - & $-/ 127.64$ & - / 1917.75* & $-/ 15.02 *$ \\
\hline$\left(\rho_{k} u_{k} k / \mu_{k}\right)=b+m_{i} f_{i}+n_{i j} g_{i j}$ & 0.89 & 1.23 & - & $-/ 46.39$ & $-/-2572.19 *$ & $-/-55.45^{*}$ \\
\hline
\end{tabular}

The reader will probably note upon examination of the correlation relationships in Tables 2 and 3 that the parameter $B_{k}$ is not a typical boundary layer parameter to include in BLT correlations. However, the authors have a specific motivation for including this term. Since $B_{k}$ is the ratio of the mean thermal velocity to sonic velocity, $\mathrm{Re}_{k} \mathrm{~B}_{\mathrm{k}} / \mathrm{M}_{\mathrm{k}}$ is effectively the inverse of a Knudsen number. The reader can confirm this by relating mean free path and viscosity in the Knudsen number and Reynolds number to equations involving the mean thermal velocity and sonic velocity. Inclusion of the $B_{k}$ value is thus intended to provide insight into the potential significance that non-continuum effects have on discrete protuberance incipient BLT.

By examining the results of the various $\mathrm{Re}_{\mathrm{k}}$ family correlations included in Tables 2 and 3, one can develop insight into the Knudsen number range that is important for incipient BLT. The linear regression coefficients shown for $\left(\mathrm{M}_{\mathrm{k}} / \mathrm{B}_{\mathrm{k}}\right)$ in Table 3 represent the Knudsen number range of significance for discrete protuberance BLT. Since the regression coefficients for $\left(\mathrm{M}_{\mathrm{k}} / \mathrm{B}_{\mathrm{k}}\right)$ shown in Table 3 are actually the inverse of the Knudsen number, we have $\left(1 / \mathrm{Kn}_{\mathrm{k}}\right) \approx(324,63,141)$ for the third, fourth and fifth correlations which are all $\mathrm{Re}_{\mathrm{k}}$ family correlations. Inverting these values yields $\mathrm{Kn}_{\mathrm{k}} \approx(0.003,0.016,0.007)$. It is commonly accepted that non- 
continuum effects can be assumed to begin playing a role in the physics of high altitude fluid dynamics for Knudsen numbers greater than 0.01. At values larger than approximately 0.05 , the engineering community typically utilizes techniques to adjust continuum based methodologies for integrated engineering parameters with similarity parameters. In Navier Stokes simulations, slip wall boundary conditions are presumed important when the Knudsen number begins exceeding approximately 0.01Based on the Knk values listed above, it appears that discrete protuberance incipient BLT occurs at Knudsen numbers not far below conventional values associated with non-continuum effects. Therefore, the results characterized in Table 3 lead to a hypothesis that non-continuum effects play a role in incipient discrete protuberance BLT onset at hypersonic conditions.

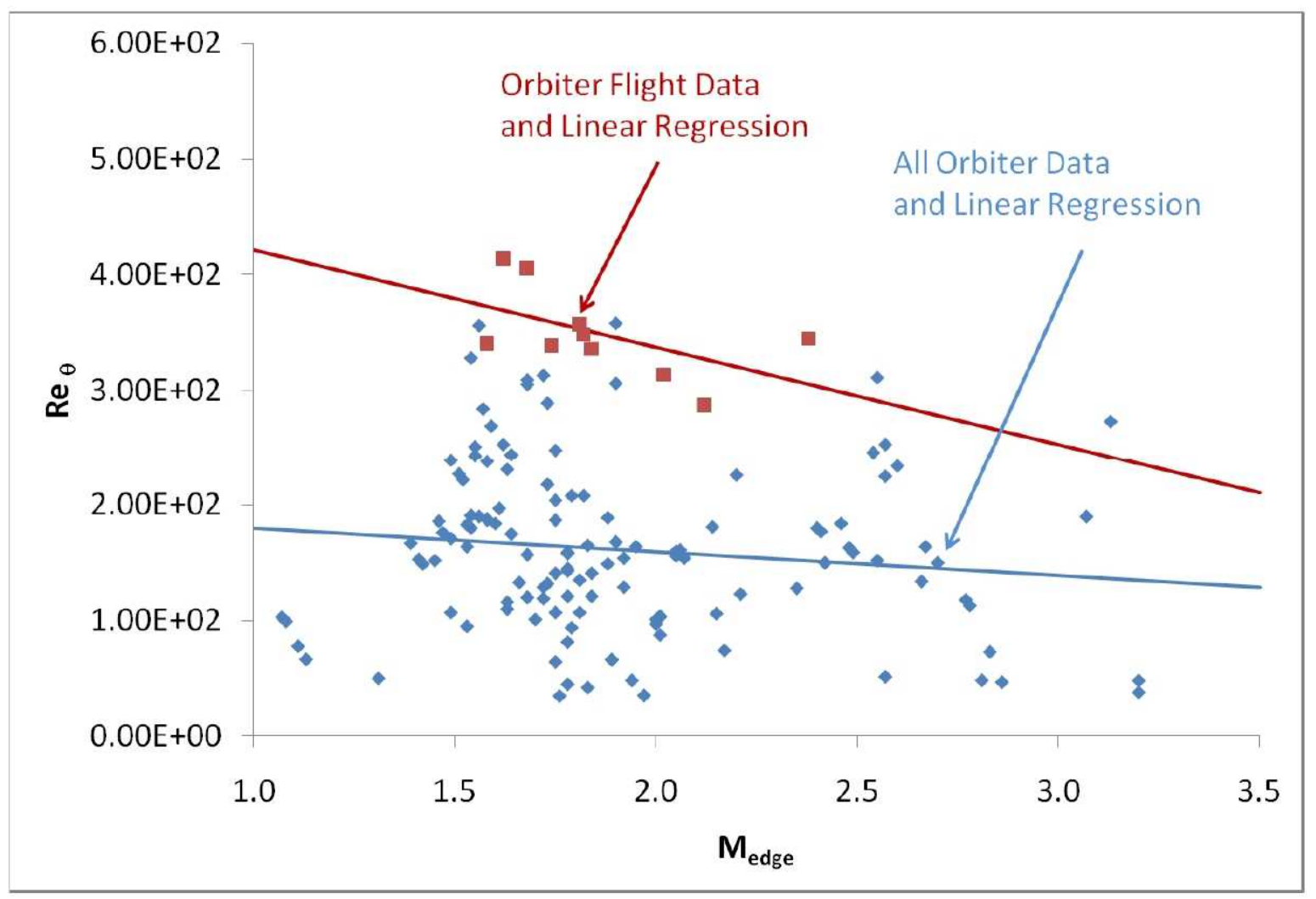

Figure 2. Orbiter Discrete Protuberance BLT Correlation of $\operatorname{Re}_{\theta}$ vs Medge. 


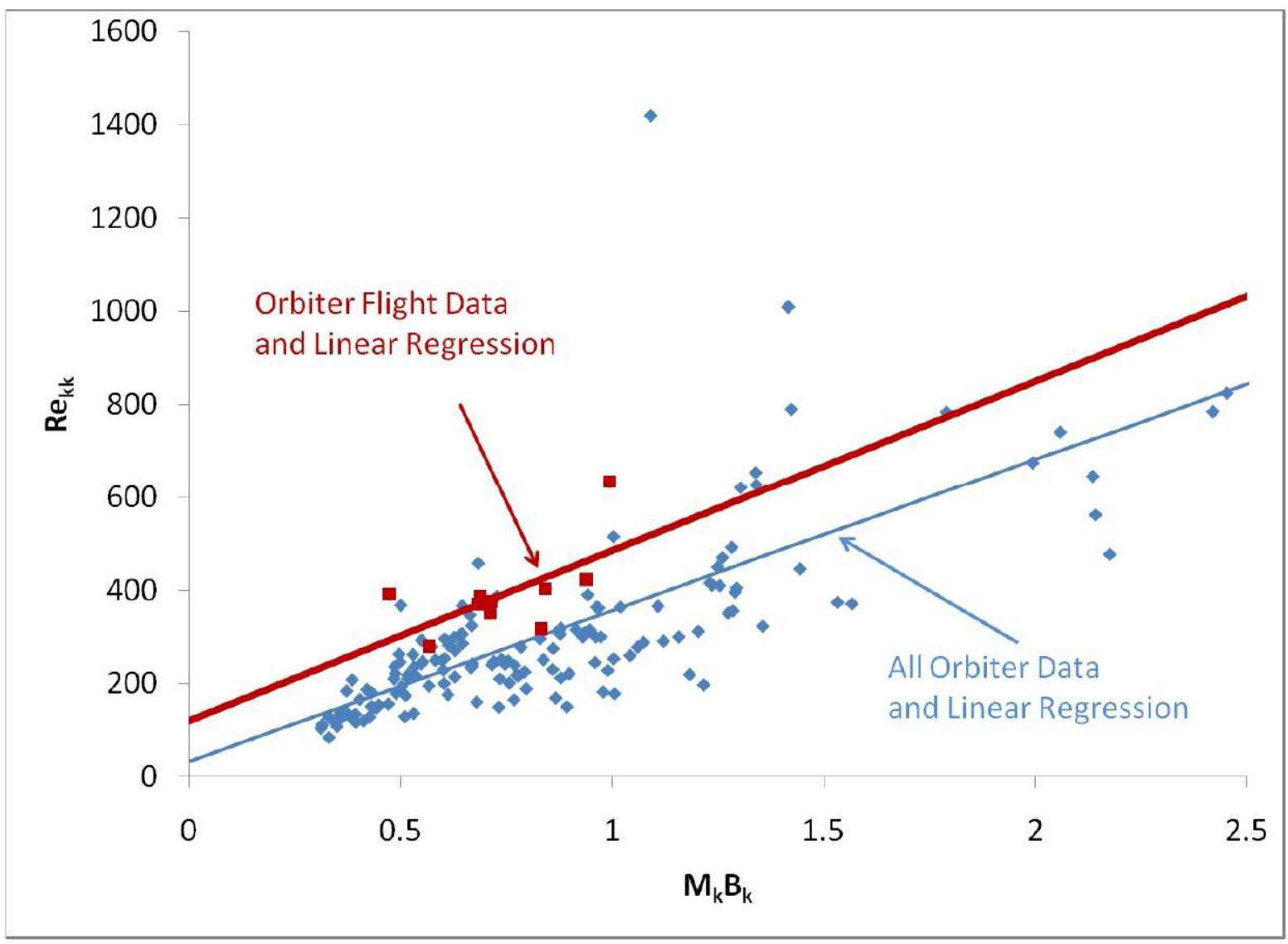

Figure 3. Orbiter Discrete Protuberance BLT Correlation of $\operatorname{Re}_{\mathrm{kk}} v_{s} \mathrm{M}_{\mathrm{k}} \mathrm{B}_{\mathrm{k}}$.

Let us now proceed on to making more general observations regarding the various correlations included in Tables 2 and 3. Note first that the simple $\operatorname{Re}_{\theta} / \mathrm{M}_{\text {edge }}$ mean value basis, shown in the first row of Tables 2 and 3, has the largest correlation nondimensional uncertainty metric, $\Delta \mathrm{F}_{4 \sigma} / \mathrm{F}_{\text {mean }}$, of any result included herein. An improved correlation uncertainty can be obtained by performing a linear regression with $\operatorname{Re}_{\theta}$ versus $M_{\text {edge, }}$ yielding roughly one-half the uncertainty. However, the regression coefficient is very low with a magnitude of 0.13 , even though the uncertainty metric has a value better, 1.08 , than many others represented in this paper. A more accurate correlation basis that falls in the $\mathrm{Re}_{\theta}$ family is included in row 1 of the power law regression results in Table 1 . That $\mathrm{Re}_{\theta}$ family power law correlation has a correlation coefficient of 0.92 , and an uncertainty metric of 1.08 .

An important observation can be made at this juncture regarding accuracy of the single parameter correlations $\mathrm{Re}_{\theta}$ and $\mathrm{Re}_{\mathrm{k} k}$. Results included in Tables 2 and 3 for these two single parameter correlations are included. A simple mean for $\mathrm{Re}_{\theta}$ and $\mathrm{Re}_{\mathrm{kk}}$ (not included in Tables 2 and 3) yield $\Delta \mathrm{F}_{4 \sigma} / \mathrm{F}_{\text {mean }}$ magnitudes of 2.16 and 2.68, respectively. Therefore, in regard to discrete protuberance BLT prediction for the Orbiter, a simple mean of $\mathrm{Re}_{\theta}$ is superior to a simple mean of Rekk. Additional comparisons can be made between the various $\mathrm{Re}_{\theta}$ and $\mathrm{Re}_{\mathrm{kk}}$ based correlations included in Tables 1, 2 and 3. Let us proceed toward evaluating the best $\operatorname{Re}_{\theta}$ and $\operatorname{Re}_{\mathrm{kk}}$ 
based correlations evaluated herein. The most accurate $\mathrm{Re}_{\mathrm{kk}}$ correlation on the Orbiter data ensemble is defined in the bottom row of Tables 2 and 3. This multiparameter linear regression of $\mathrm{Re}_{\mathrm{kk}}$ using boundary layer parameters has a regression coefficient of 0.89 and a $\Delta \mathrm{F}_{95} / \mathrm{F}_{\text {mean }}$ magnitude of 1.23 . This result is slightly better than the best power-law correlation of $\mathrm{Re}_{\mathrm{kk}}$ included in Table 1 which has a $\Delta \mathrm{C}_{95} / \mathrm{C}$ magnitude of 1.30 . However, the difference in correlation forms (e.g. linear versus power law) perhaps makes this small difference less significant. The best $\operatorname{Re}_{\theta}$ based correlation is identified in the first row of Table 1 , and is also a power-law correlation. The best $\operatorname{Re}_{\theta}$ based correlation of the Orbiter data ensemble has a regression coefficient of 0.92 and a $\Delta \mathrm{C}_{95} / \mathrm{C}$ magnitude of 1.05 . Therefore, the statistical evaluation values for the $\operatorname{Re}_{\theta}$ basis are better than for the $\operatorname{Re}_{\mathrm{k}}$ basis. The conclusion is that the $\operatorname{Re}_{\theta}$ family correlation frameworks are superior to the $\mathrm{Re}_{\mathrm{k}}$ family correlations on the Orbiter configuration whether a simple mean, single parameter linear regressions or power-law correlations are utilized. Care should be takend in applying this conclusion to other vehicles, however. Configurations other than the Orbiter may yield accuracy and uncertainty trends that result in different conclusions, conclusions which can only be established by open minded consideration of multiple correlation parameters.

The information provided earlier in this section has been used to illustrate an example of how quantitative evaluations of statistically significant BLT onset data can be used to draw concrete conclusions regarding the accuracy and uncertainty of different correlations. The primary conclusion of this assessment is that $\operatorname{Re}_{\theta}$ family empirical correlations for Orbiter configuration discrete protuberance BLT prediction are superior to $\mathrm{Re}_{\mathrm{k}}$ family correlations. For the Orbiter configuration, this conclusion is true whether a simple mean or power-law regressions form the correlation basis. The authors recommend that this conclusion be carefully considered with respect to current and future efforts, as well as efforts involving study of historical data sets. Consideration should also be given to application of the tenet utilized by the Orbiter RTF team that developed BLT correlations for the Space Shuttle program whereby multiple parameter spaces be considered and quantitatively evaluated in order to obtain the most accurate empirical correlation possible. Adherence to a focus on correlating BLT onset data with a single parameter space, which would be contrary to a tenet practiced by the Orbiter team, would deny the broader community and the programs providing support to an effort the option of understanding which empirical correlations are the most relevant to a given situation.

Before moving on to a brief discussion summarizing the results presented in this paper and looking to the future, a few more observations regarding the engineering role of empirical BLT correlations shall be provided. It should be noted that it is not necessarily sufficient as an engineer to establish the most accurate prediction basis able to support a decision in the allotted time. For example, earlier in this section, a conclusion has been made that $\mathrm{Re}_{\theta}$ based empirical correlations are superior to $\mathrm{Re}_{\mathrm{k}}$ correlations for the prediction of discrete protuberance BLT on the Orbiter configuration. Because these correlations are, after all, regression based curve fits of 
relevant data we lack the ability to gain detailed physical insight into the mechanisms and processes that are at work. When faced only with methods that lack a detailed physical rationale, it is prudent engineering practice to have more than one independent method to support a decision making process. This is particularly true in the case of human spaceflight missions and designs. For this reason, the Orbiter RTF entry aerothermodynamics team implemented both $\operatorname{Re}_{\theta}$ and $\mathrm{Re}_{\mathrm{k}}$ based discrete protuberance BLT correlations to support the Space Shuttle program.

\section{Discussion}

Reference other paper session topics, AF/ARMD Hypersonic Center at Texas S\&M, AF/ARMD-FAP Foundational Research Plan all these being indication of interest and recent investments. Hopeful future, one that if successful will provide higerh fidelity capabilities for BLT prediction that are practical and provide more accurate and more precise BLT prediction capabilities.

\section{Summary and Conclusions}

\section{AAA}

\section{References}

Need to build in comments on Reshotko (Wright 41 - altitude correlation) and Pate (Berry 32 - tunnel noise) and Lin (DoD empirical correlation)

Campbell, C.H., Anderson, B.P., King, R.A., Kegerise, M.A., Berry, S.A., Horvath, T.J., "Roles of Engineering Correlations in Hypersonic Entry Boundary Layer Transition Prediction," AIAA 2010-XXXX.

Campbell, C.H., Garske, M.T., Kinder, G., Berry, S.A., “Orbiter Entry Boundary Layer Flight Testing", AIAA 2008-635.

Bertin, J.J., Schneider, S.P., Campbell, C.H., "A Flight-Testing Proposal on RoughnessInduced Boundary-Layer Transition on the Space Shuttle Orbiter", NASA White Paper, November 14, 2006.

Berry, S.A., King. R.A., Kegerise, M.A., Wood, W., McGinley, C., Anderson, B.P., "Updated to Orbiter Boundary Layer Transition Prediction Tool," AIAA 2010-XXXX, January 2010.

Campbell, C.H., et.al, “Orbiter Return to Flight Entry Aeroheating”, AIAA 2006-2917, June 2006. 
Spanos, T., Kinder, G., Campbell, C.H., Andress, J., "Boundary Layer Transition Flight Experiment Thermal Protection System and Instrumentation Modifications", AIAA 2010-XXXX, January 2010.

Berry, S.A., Horvath, T.J., Cassady, A.M., Kirk, B.S., Wang, K.C., Hyatt, A.J., "Boundary Layer Transition Results from STS-114", AIAA 2006-2922.

McGinley, C.B., Berry, S.A., Kinder, G.R., Barnwell, M., Wang, K.C., Kirk, B.S., "Review of Orbiter Flight Boundary Layer Transition Data", AIAA 2006-2921.

King, R.A and Vaughn, M.P., "Turbulent Wing-Leading-Edge Correlation Assessment for the Shuttle Orbiter", NASA TM-2009-215949 (DRAFT)

King, R.A., Kegerise, M.A., and Berry, S.A., "Version 2 of the Protuberance Correlations for the Shuttle-Orbiter Boundary Layer Transition Tool”, NASA-TP2009-XXXXX (In Review)

Bartlett, E.P., Abbett, M.J., Nicolet, W.E., and Moyer, C.B., "Improved Heat-Shield Design Procedures for Manned Entry Systems, Part II - Application to Apollo", June 22, 1970, NASA CR-108689.

For NASP:

Lau, K. Y., Hypersonic Boundary-Layer Transition: Application to High-Speed Vehicle Design, J. Spacecr. Rockets 45(2): 176-183.

For Hyper-X flight data:

Berry, S., Daryabeigi, K., Wurster, K., Bittner, R., "Boundary Layer Transition on X43A," AIAA Paper 2008-3736, June 2008.

For HyBoLT:

Berry, S.; Chen, F.; Wilder, M.; and Reda, D.; "Boundary Layer Transition

Experiments in Support of the Hypersonics Program," AIAA-2007-4266, 39th

AIAA Thermophysics Conference, Miami, FL, June 25-28, 2007.

Poll, D.I.A., "Boundary Layer Transition on the Windward Face of the Space Shuttle During Re-Entry,” AIAA paper 85-0899, June 1985. 